\title{
Bilateral Striatal Necrosis after Sydenham's Chorea in a 7-Year-Old Boy: A 2-Year Follow-Up
}

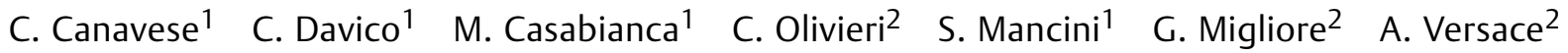 \\ A. Tocchet ${ }^{1}$ B. Vitiello ${ }^{1}$
}

${ }^{1}$ Department of Pediatric Neurology, University of Turin, Children Hospital Regina Margherita, Turin, Italy

2 Department of Pediatric Emergency, Children Hospital Regina

Margherita, Turin, Italy

Neuropediatrics 2018;49:209-212.

\begin{abstract}
Address for correspondence C. Davico, MD, Department of Pediatric Neurology, University of Turin, Children Hospital Regina Margherita, Piazza Polonia 94, 10100 Turin, Italy (e-mail: caiadav@fastwebnet.it).
\end{abstract}

\begin{abstract}
Keywords

- bilateral striatal necrosis

- Sydenham's chorea

- movement disorder

- dystonia

- group A streptococcus

Child bilateral striatal necrosis (BSN) is a rare and etiologically heterogeneous condition. An association with group A streptococcus (GAS) infection was previously reported in two cases of BSN in infancy and early childhood. We here report on a 7-year-old boy who developed chorea and dystonia 20 days after symptomatic recovery from Sydenham's chorea. Repeated brain magnetic resonance imaging scans, obtained before, soon after the onset of the post-Sydenham symptoms, and 1 year later were consistent with an evolution from bilateral striatal microbleeding to necrosis, and consequently reduced basal ganglia volume and enlargement of the frontal horns. No support was found for other possible autoimmune, infectious, metabolic, toxic or genetic etiologies for BSN. Prednisone treatment was instituted and continued for 1 year. Two years after the onset of the post-Sydenham symptoms, the child, although much improved, still has generalized dystonic-choreic movements. This case confirms and extends into school age, the link between GAS and BSN.
\end{abstract}

\section{Introduction}

Bilateral striatal necrosis (BSN) is a rare condition pathologically characterized by insult to the neostriata (putamen and caudate), ultimately leading to cellular necrosis. ${ }^{1,2}$ It presents clinically with dystonic movements and is accompanied by specific neuroimaging abnormalities. ${ }^{1}$ Permanent neurological sequelae are common, even though full recovery has been reported. ${ }^{2}$ The etiology of BSN is heterogeneous, including viral and mycoplasma infections, mitochondrial disorders, autoimmune encephalitis, genetic factors, hypoglycemia, and exposure to exogenous or endogenous toxins. ${ }^{1-3}$

Group A streptococcus (GAS) can be involved in various neurological disorders caused by aberrant immune responses to its antigens, with a consequent autoimmune disease. The spectrum of post-GAS disease of the central nervous system includes movement disorders (chorea, tics, dystonia, and Parkinsonism), due to involvement of basal ganglia.

received

July 26, 2017

accepted after revision

December 5, 2017

published online

February 22, 2018

Previously, two cases of BSN consequent to GAS infection were reported, one in a 14-month old infant and the other in a 4 -year-old child. ${ }^{4}$ In these cases, the clinical presentation included dystonia, but not chorea.

We here describe a case of a 7-year-old boy first diagnosed with Sydenham's chorea (SC), who later progressively developed a generalized choreodystonic syndrome associated with BSN.

\section{Case Report}

A 7-year-old boy presented to our hospital for worsening of involuntary movements of the limbs that had started 10 days before, together with unstable walking and dysgraphia. There were repetitive, chaotic, asynchronous involuntary movements of the four limbs, tongue impersistence, and dysgraphia (see - Video 1). Past medical history was unremarkable, except for recurrent pharyngitis.

(C) 2018 Georg Thieme Verlag KG Stuttgart - New York
DOI https://doi.org/ 10.1055/s-0037-1618590. ISSN 0174-304X. 


\section{Video 1}

Repetitive, chaotic, asynchronous involuntary movements of the four limbs, tongue impersistence, and dysgraphia at onset. Online content including video sequences viewable at: www.thieme-connect. com/products/ejournals/html/10.1055/s-00371618590.

A clinical diagnosis of SC was supported by laboratory testing showing high antistreptolysin $O$ titer $(643 \mathrm{IU} / \mathrm{mL})$ and high level of antideoxyribonuclease antibody $(1274 \mathrm{IU} / \mathrm{mL}$ ). Nasopharyngeal swab was positive for GAS $\beta$-hemolytic. The other laboratory tests (antinuclear antibodies, lupus anticoagulant, anticardiolipin antibodies, $\beta 2$-glycoprotein I antibodies) as well as cardiac echography were normal.

The boy was treated with oral prednisone $2 \mathrm{mg} / \mathrm{kg} /$ day, and prophylaxis with benzathine penicillin was started. Due to the extremely intense movement disorder, oral haloperidol $1 \mathrm{mg} /$ day was added and tapered off over 15 days. He progressively improved and reached full symptomatic remission in 10 days.

At this time, brain magnetic resonance imaging (MRI) showed bilateral T2 hyperintensity, without restricted diffusion on diffusion-weighted images, and mild T1 hyperintensity in the caudate nuclei and anterior portion of putamina (-Fig. 1A, B). These images were interpreted as microbleeding following vasculitis in rheumatic chorea. ${ }^{5}$

Metabolic investigations (lactate and pyruvate, ammonia, amino acids, organic acid, acylcarnitine, carboxyhemoglobin) and testing for thrombophilia were normal. No evidence was found for possible carbon monoxide poisoning.

After being in remission and completely asymptomatic for 20 days, neurological symptoms reappeared with different features: choreic movements were associated with dystonia and stiffness, mainly involving the legs (see - Video 1 ). A new brain MRI was performed, showing a mild reduction in previously described $\mathrm{T} 2$ hyperintensity and an increase in T1 hyperintensity in the caudate nuclei and the anterior portion of putamina (-Fig. 1C, D), suggesting an evolution toward BSN. No Kayser-Fleischer rings were visible. Tests of copper metabolism, thiamin, and antibodies to Mycoplasma pneumoniae were normal.

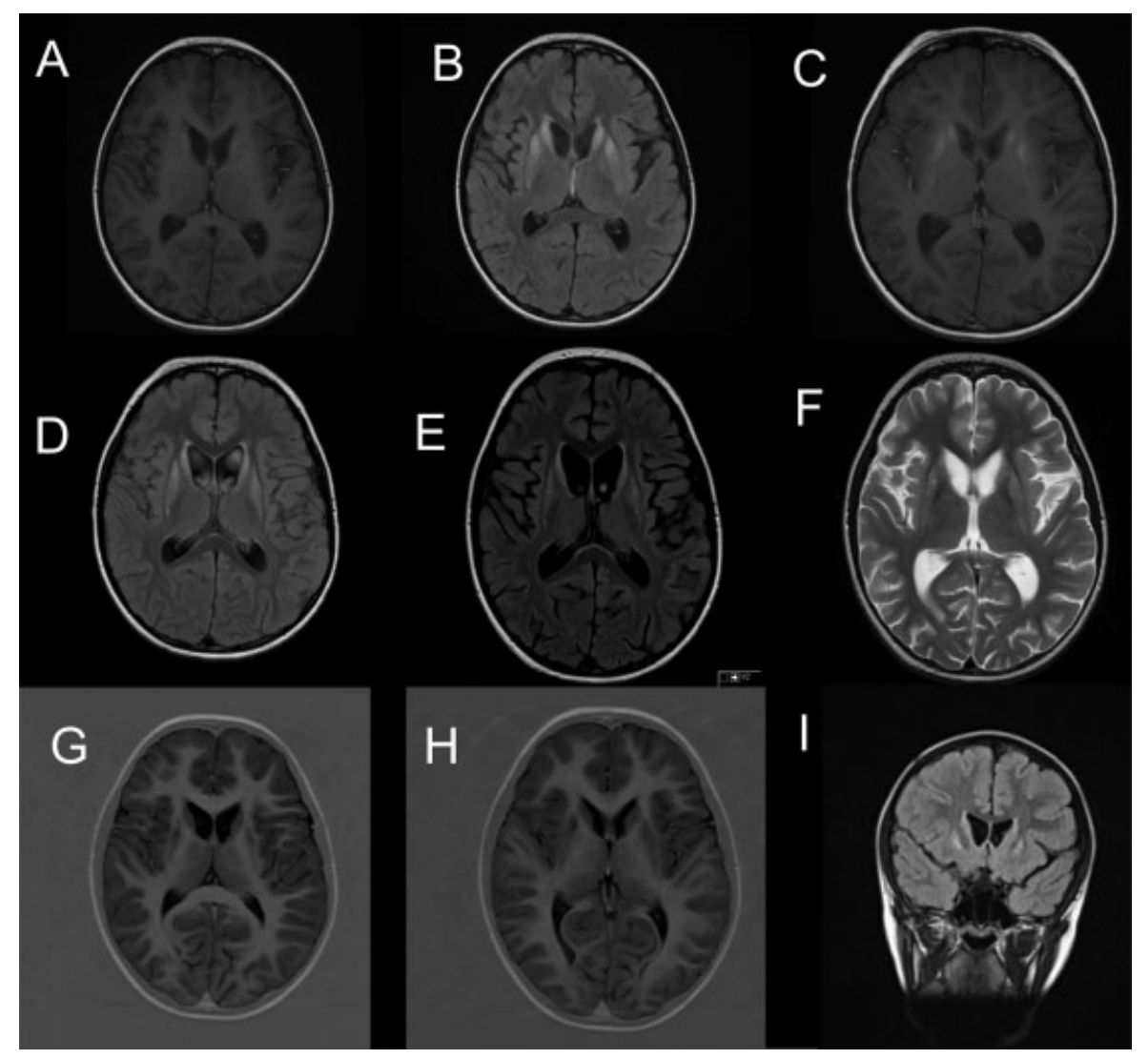

Fig. 1 (A) First magnetic resonance imaging (MRI) (25 days after onset), axial T1-weighted image showing mild hyperintensity in caudate nuclei and anterior portion of putamina. (B) First MRI, axial fluid-attenuated inversion recovery (FLAIR) image showing hyperintensity in caudate nuclei and anterior portion of putamina. (C) Third MRI (1 month and 15 days after onset), axial T1-weighted image showing an increase in hyperintensity in the caudate nuclei and anterior portion of putamina. (D) Fourth MRI, axial FLAIR showing hyperintensity in the caudate nuclei and anterior portion of putamina. (E, F) Fifth MRI ( 3 months and a half after onset), axial FLAIR and T2-weighted images showing hyperintensity in the caudate nuclei and anterior portion of putamina with reduced volume of basal nuclei and enlarged frontal horns. (G, H) Sixth MRI ( 2 years after onset), axial T1-weighted image showing cavitation in caudate nuclei and anterior portion of putamina. (I) Seventh MRI, coronal flair image showing hyperintensity in caudate nuclei and anterior portion of putamina. 
A brain computed tomography excluded cerebral calcifications. Cerebrospinal fluid (CSF) showed normal cell count, glucose, lactate, amino acids, and proteins. CSF polymerase chain reaction for group A streptococcus and M. pneumoniae was negative. The CSF autoantibodies panel for autoimmune encephalitis, including AMPA, GABA(b), mGluR1, mGluR5, LGI1, Caspr2, NMDAR, and D2R were negative. Analysis of the genetic panel for striatal bilateral necrosis was negative for the following genes: ADAR, GCCDH, NUP62, SLC19A3, SLC25A19, TPK1, TUBB4A. Antistreptolysin O antibodies titer was $310 \mathrm{IU} / \mathrm{mL}$ and antideoxyribonuclease antibodies titer was $620 \mathrm{IU} / \mathrm{mL}$.

The patient was treated with intravenous immunoglobulin (in consideration of a postulated postinfectious GAS autoimmune mechanism), but with negligible response. He was then given pulse intravenous methylprednisolone, starting with $30 \mathrm{mg} / \mathrm{kg} /$ day, gradually reduced in the following days, with a considerable improvement, but not complete regression, in the involuntary movements (see - Video 1 ). Oral methylprednisolone was then substituted for prednisone and this treatment was continued for about 1 year. Additional treatment included therapy with tetrabenazine, oral supplementation of thiamine and biotin, and physical rehabilitation.

Brain MRI performed 2 months later showed vanishing of T1 hyperintensity (previously described in the caudate nuclei and the anterior portion of putamina) but $\mathrm{T} 2$ hyperintensity in the same areas persisted unmodified ( - Fig. 1 $\mathbf{E}, \mathbf{F})$; the volume of basal nuclei was reduced and the frontal horns were enlarged. These images confirmed a stabilization of the necrotic process.

Currently, 2 years after the onset, the child is in a stable clinical condition, with a persistent, although attenuated, generalized dystonic-choreic syndrome. There has been no cognitive and behavioral deterioration. He receives ongoing thiamine, biotin, and benzathine penicillin prophylaxis. Trihexifenidilo was briefly tried, but caused blurred vision and no significant benefit. The latest brain MRI, 2 years after onset, confirmed the reduction in basal nuclei volume with signs of cavitation ( - Fig. 1 G-I).

\section{Discussion}

To our knowledge, this is the first report of a case of BSN in a school-age child that can be etiologically linked to GAS infection. After developing SC, this patient clinically recovered and was asymptomatic for 20 days before presenting with generalized dystonic-choreic movements and brain imaging evidence of BSN.

SC is considered the prototype autoimmune disease of central nervous system triggered by a streptococcal infection. ${ }^{5-10}$

BSN following streptococcal infection is a very rare complication and only two cases had been previously reported. ${ }^{4}$ These patients were very young (14-month and 4-year old, respectively), and both presented with dystonia, but not chorea. It can be speculated that the age when the immunological basal ganglia insult occurs may influence the resulting clinical manifestation. For example, an infantile or early child- hood insult may result in a dystonic outcome, whereas a later childhood disease may result in chorea.

In post-GAS disorders affecting central nervous system, brain MRI is usually normal, except for unspecific inflammatory changes in the basal ganglia. ${ }^{11}$ In post-GAS or a specific postinfectious extrapyramidal movement disorder, basal ganglia can show MRI signs consistent with inflammation and seldom necrosis. ${ }^{12,13}$

In BNS, brain MRI shows initial T2 and fluid-attenuated inversion recovery (FLAIR) hyperintensity of swollen neostriata and a subsequent hypointensity on T1-weighted images due to progressive atrophy. ${ }^{14}$

When our patient first developed SC, brain MRI showed bilateral T2 hyperintensity of basal ganglia, which were interpreted as consequence of microbleeding due to vasculitis in rheumatic chorea. These lesions progressively evolved into necrosis, as evidenced by MRI signs of atrophy.

Extensive and repeated testing ruled out alternative explanations, including other infections, metabolic, toxic, or genetic causes. The possibility of an autoimmune encephalitis was considered, but ruled out by negative CSF antiNMDAR and D2 antibodies tests.

A considerable reduction in choreoathetotic movements and dystonia was achieved on intravenous steroids therapy, thus supporting an inflammatory/autoimmune genesis of the pathological process. The symptomatic course was consistent with the pathological process: the initial classic choreic movements eventually abated, but to be then followed, after a 20-day asymptomatic period, by more complex dystonic postures and movements. Two years later, though much improved, he still presents with dystonia, which can be now considered stable and permanent. It is noteworthy that there are no other neurological or cognitive deficits. Thus, the clinical, laboratory, brain imaging, and treatment data are all consistent with a diagnosis of post-GAS BSN.

\section{Conclusion}

This case shows that BSN can occur consequent to SC in schoolage children, and provides further evidence that GAS infection can, in rare cases, trigger a cascade of events resulting in permanent basal ganglia damage and neurological sequelae.

\section{Conflict of Interest}

None.

\section{References}

1 Tonduti D, Chiapparini L, Moroni I, et al. Neurological disorders associated with striatal lesions: classification and diagnostic approach. Curr Neurol Neurosci Rep 2016;16(06):54

2 Nandish S, Khan R, Connolly DJ, Rittey CD, Mordekar SR. Reversible parainfectious bilateral "striatal necrosis". Pediatr Neurol 2012;46(01):51-53

3 Tzoulis C, Vedeler C, Haugen M, et al. Progressive striatal necrosis associated with anti-NMDA receptor antibodies. BMC Neurol 2013; 13:55

4 Dale RC, Church AJ, Benton S, et al. Post-streptococcal autoimmune dystonia with isolated bilateral striatal necrosis. Dev Med Child Neurol 2002;44(07):485-489 
5 Giedd JN, Rapoport JL, Kruesi MJ, et al. Sydenham's chorea: magnetic resonance imaging of the basal ganglia. Neurology 1995;45(12):2199-2202

6 Punukollu M, Mushet N, Linney M, Hennessy C, Morton M. Neuropsychiatric manifestations of Sydenham's chorea: a systematic review. Dev Med Child Neurol 2016;58(01):16-28

7 Dale RC. Autoimmunity and the basal ganglia: new insights into old diseases. QJM 2003;96(03):183-191

8 Gewitz MH, Baltimore RS, Tani LY, et al; American Heart Association Committee on Rheumatic Fever, Endocarditis, and Kawasaki Disease of the Council on Cardiovascular Disease in the Young. Revision of the Jones Criteria for the diagnosis of acute rheumatic fever in the era of Doppler echocardiography: a scientific statement from the American Heart Association. Circulation 2015;131(20):1806-1818

9 Dale RC, Church AJ, Surtees RA, Thompson EJ, Giovannoni G, Neville BG. Post-streptococcal autoimmune neuropsychiatric disease presenting as paroxysmal dystonic choreoathetosis. Mov Disord 2002;17(04):817-820
10 Dale RC, Church AJ, Cardoso F, et al. Poststreptococcal acute disseminated encephalomyelitis with basal ganglia involvement and auto-reactive antibasal ganglia antibodies. Ann Neurol 2001; 50(05):588-595

11 Dale RC. Post-streptococcal autoimmune disorders of the central nervous system. Dev Med Child Neurol 2005;47(11):785-791

12 Karagulle Kendi AT, Krenzel C, Ott FW, Brace JR, Norberg SK, Kieffer SA. Poststreptococcal dystonia with bilateral striatal enlargement: MR imaging and spectroscopic findings. AJNR Am J Neuroradiol 2008;29(07):1276-1278

13 Zevit N, Steinmetz A, Kornreich L, Straussberg R. Acute infantile bilateral striatal necrosis: single-photon emission computed tomography (SPECT) imaging and review. J Child Neurol 2007;22(10): 1222-1226

14 Livingston JH, Lin JP, Dale RC, et al. A type I interferon signature identifies bilateral striatal necrosis due to mutations in ADAR1. J Med Genet 2014;51(02):76-82 